\title{
JAPÓN: UN PAÍS \\ DE CLASE MEDIA \\ EN TRANSICIÓN
}

Ulises Granados*

RESUMEN: Mientras que ciertos elementos sentaron las bases de la clase media japonesa en la posguerra, su consolidación y los cambios sufridos en la sociedad desde la década de 1990 dieron paso a una clase media adelgazada y precaria, que ha resentido la desigualdad económica, las alteraciones del patrón laboral y las tendencias marcadas por las reformas estructurales.

\section{sose \\ JAPAN: A MIDDLE CLASS COUNTRY IN TRANSITION}

ABSTRACT: While certain elements laid the foundations of the Japanese middle class in the postwar period, its consolidation and the changes undergone in society since the 1990s gave way to a thinned and precarious middle class, which has suffered from economic inequality, alterations in the labor pattern and the tendencies marked by structural reforms.

PALABRAS CLAVE: Abenomics, posguerra, transición KEY WORDS: abenomics, postwar, transition.

RECEPCIÓN: 21 de mayo de 2017.

APROBACIÓN: 15 de febrero de 2018.

DoI: $10.5347 / 01856383.0128 .000292933$

* Programa de Estudios Asia Pacífico PEAP, ITAM. 
Se prohíbe su reproducción total o parcial por cualquier medio, incluido electrónico, sin permiso previo y por escrito de los editores. 


\section{JAPÓN: UN PAÍS \\ DE CLASE MEDIA \\ EN TRANSICIÓN}

\section{Introducción}

\section{Muchas veces se presenta a Japón} como un país de clase media por excelencia, una clase media que emergió en el período posterior a la Segunda Guerra Mundial. ${ }^{1}$ Hace más de treinta años, William Kelly identificó esta imagen estereotipada de la familia japonesa de ingresos medios: una familia nuclear integrada por el padre ocupado en su trabajo, la madre dedicada al hogar y uno o dos hijos "samuráis" en edad escolar que perfeccionan sus conocimientos en academias vespertinas. ${ }^{2}$ Esta familia ideal era el modelo de una sociedad japonesa en la que el padre tenía un empleo de tiempo completo y de por vida, la educación pública permeaba toda la sociedad como llave para obtener un trabajo basado en la meritocracia y aprobar los exámenes de admisión a las universidades. Este fue el modelo aspiracional forjado para una cultura japonesa moderna y urbanizada que se levantó de las cenizas de la guerra entre 1945 y 1990.

Uno de los indicadores con los que se evalúa la percepción de la clase social en el país es el sondeo de la Oficina del Gabinete, llamado Estratificación Social y Movilidad (Social Stratification and Mobility,

${ }^{1}$ Ezra F. Vogel, The salary man and his family in a Tokyo suburb, 1971, Berkeley, University of California Press, p. 4.

${ }^{2}$ William W. Kelly, "Rationalization and nostalgia: Cultural dynamics of new middleclass Japan”, American Ethnologist, vol. 134-4 (1986), pp. 603-618. 
SSM), que la Sociedad Sociológica de Japón realizó por primera ocasión en 1955 y continuó aplicando hasta 2005, a fin de cuantificar el estatus social de la población, la movilidad social y la percepción de la población sobre clase social y desigualdad. Desde el comienzo, la encuesta reveló el aumento en la percepción de un gran porcentaje de la población (casi el 70\% de los encuestados a mediados de la década de 1950) de que pertenece a la clase media, ya sea media alta, media o media baja. ${ }^{3}$ Sin embargo, esta percepción, que alcanzó alrededor del 90\% a mediados de la década de 1970, continúa siendo objeto de controversias.

En realidad, la narrativa de este modelo de clase media, reforzada por el régimen político y los medios masivos de comunicación desde inicios de la posguerra, ${ }^{4}$ dista mucho de ser cierta, no solo ahora, sino desde la década de 1980, y ha sido objeto de fuertes críticas ya desde la década siguiente, al identificar en Japón una reproducción de la desigualdad social muy similar a la de otras "democracias capitalistas". 5 Sugimoto afirma que, en realidad, el país siempre ha mantenido una estratificación social más compleja que incluye una diversidad subcultural y una competencia entre clases sociales. ${ }^{6}$ En la década de 1990, Kelly ${ }^{7}$ había calculado que más del $50 \%$ de las mujeres tenían que trabajar para ayudar a sostener a la familia y que apenas poco más de la mitad de los egresados de la educación media superior podían inscribirse en una universidad, cuanto menos una de prestigio, y que solo entre $30 \%$ y $40 \%$ de los trabajadores tenían un empleo de tiempo completo y seguridad laboral vitalicia.

${ }^{3}$ Kunio Odaka, "The middle classes in Japan”, The Sociological Review, 10/S1 (1962), p. 41; William W. Kelly, "At the limits of new middle-class Japan: Beyond 'mainstream consciousness"”, en Oliver Zunz, Leonard Shoppa y Nobuhiro Hiwatari (comps.), Social contracts under stress, 2004, Nueva York, Russell Sage Foundation, pp. 233-235.

${ }^{4}$ David Chiavacci, "From class struggle to general middle-class society to divided society: Societal models of inequality in postwar Japan", Social Science Japan Journal, 11/1 (2008), pp. 10-13.

${ }^{5}$ David H. Slater, "The making of Japan's new working class: Freeters and the progression from middle school to the labor market", The Asia-Pacific Journal, 8/1 (2010), p. 5.

${ }^{6}$ Yoshio Sugimoto, An introduction to Japanese society, 1997, Cambridge, Cambridge University Press, p. ix.

${ }^{7}$ Kelly, "Rationalization and nostalgia", p. 605. 
De cualquier forma, la aproximación más segura al tema, de acuerdo con estudios previos, es asumir que un alto porcentaje de la población japonesa se ha sentido parte de una nutrida clase media relativamente homogénea. Ahora, esto terminó y dio paso a una sociedad cada vez más desigual.

Y es que la tesis de la sociedad relativamente igualitaria de ese país tiene bases cuantitativas. Según estimaciones de la OCDE, en $1976^{8}$ Japón tenía un coeficiente de Gini $^{9}$ de 0.316 , más bajo que el de Alemania Occidental, Estados Unidos y el promedio de la OCDE; sin embargo, para 2014 el índice de Japón fue de 0.33 , ya por arriba de Suecia (0.28) Alemania (0.29) y del promedio de la OCDE (0.31), aunque todavía por debajo de Estados Unidos (0.39). ${ }^{10}$ De aquí se desprende que en cuarenta años, se deterioró en Japón la distribución de los ingresos, que es el principal elemento de la percepción de la desigualdad social. En otras palabras, Japón fue durante varias décadas un país con poca desigualdad, pero ahora está cambiando. Algunos autores han señalado que el nivel de desigualdad en Japón fue particularmente marcado a finales de la década de 1980, con índices de Gini de 0.421 en $1989 .{ }^{11}$

TABLA 1

Coeficiente de Gini, países seleccionados de la OCDE (1976 y 2014)

\begin{tabular}{llll}
\hline \multicolumn{1}{c}{ País } & \multicolumn{1}{c}{1976} & 2014 \\
\hline Japón & 0.31 & 0.33 & \\
Suecia & 0.30 & 0.28 & \\
Alemania & 0.38 (Alemania Occidental) 0.29 & \\
Estados Unidos & 0.38 & 0.39 & \\
Promedio OCDE & 0.35 & 0.31 \\
\hline
\end{tabular}

Fuente: Rebick, op. cit., p. 181; OCDE, op. cit.

${ }^{8}$ Marcus Rebick, "The myth of the middle-mass society: Inequality and emerging divisions in Japanese society", Conferencia sobre la clase media en Asia: Taiwán y otras perspectivas, Oxford, St. Anthony College, 27 de junio de 2004, en $<$ http://www.rchss.sinica.edu.tw/files/ publish/1150_e611d2db.pdf>, consultado el 15 de febrero de 2018.

${ }^{9}$ El coeficiente de Gini es un indicador de la desigualdad de los ingresos o la distribución de la riqueza.

${ }^{10}$ OECD, "Inequality", 2014, en <http://www.oecd.org/social/inequality.htm>, consultado el 15 de febrero de 2018.

${ }^{11}$ Sugimoto, op. cit., p. 10. 
Si en los primeros cuarenta años de la posguerra Japón se mostró como un país con una engrosada clase media, a partir de la década de 1990 la historia del "país de la clase media" ha perdido sustento y se ha revelado una sociedad con graves diferencias sociales reflejadas en el ingreso y mucho más marcadas. Japón es ahora un país menos igualitario que en las primeras décadas de la posguerra. Es al mismo tiempo causa y consecuencia de varios cambios socioeconómicos que afectan a la sociedad. Así pues, ¿Japón ha dejado finalmente de ser una sociedad de clase media?

\section{Consolidación del país de la clase media}

El fenómeno de la consolidación en el Japón del siglo XX de una "nueva clase media", resultado de la modernización económica del país y claramente diferente de una "vieja clase media", integrada por comerciantes y empresarios y más característica de la era Meiji (1868-1912), se percibe desde la década de 1920. Esta categoría social coherente estaba ya integrada por intelectuales, profesionales y, sobre todo, asalariados. ${ }^{12}$ Después de la Guerra del Pacífico, esta clase media comenzó a engrosarse con la recuperación económica, primero inducida por las fuerzas de ocupación y posteriormente por políticas selectivas de desarrollo.

Luego de diez años de reconstrucción para recuperarse de la devastación de la guerra, el gobierno japonés inició un exitoso despegue económico, en el que se distinguen dos periodos principales, el primero de 1955 a 1965 (con tres auges) y el segundo de 1966 a 1972, que es una etapa de crecimiento sostenido hasta la primera crisis del petróleo. Sin embargo, para efectos de crear las bases de una clase media, el periodo inmediato de la posguerra, el de la ocupación, reviste especial importancia.

Desde 1946, la recuperación económica, guiada por las fuerzas de ocupación estadounidenses encabezadas por el general Douglas

${ }^{12}$ Louise Young, "Marketing the modern: Department stores, consumer culture, and the new middle class in interwar Japan", International Labor and Working-Class History, 55 (1999), p. 61 . 
MacArthur, se materializó por medio de tres importantes reformas: la reforma agraria, la disolución de los zaibatsu (conglomerados monopólicos industriales) y una efectiva reforma laboral. ${ }^{13}$ Por un lado, mediante la reforma agraria alrededor del 37.5\% de las tierras cultivables de Japón cambiaron de manos por obra de las compras del gobierno. Por el otro, con la disolución de los zaibatsu (en 1947 unas 83 empresas fueron catalogadas como zaibatsu, de las cuales 28 eran empresas familiares) se logró disminuir la concentración del sector industrial. Más adelante, estas empresas disueltas (como Mitsui, Mitsubishi o Japan Steel Corporation) resurgieron con otro modelo, el llamado keiretsu (modelo empresarial más horizontal organizado alrededor de un banco), que fomentó la competencia entre las industrias. Al mismo tiempo, como parte de la reforma laboral, entre 1945 y 1947 se promulgaron leyes para estimular la actividad de los sindicatos y mejorar las condiciones de los asalariados.

Así, redistribución de la tierra, fomento a la competencia económica en diversos sectores de la economía y relaciones más claras entre el sector laboral y patronal sentaron las bases del crecimiento en el país, y con ello, de la clase media de la posguerra. Otras importantes condiciones que permitieron el crecimiento económico de Japón en la posguerra fueron un aumento demográfico, particularmente en la zona metropolitana de Tokio $^{14}$ (aunque hubo una ligera disminución de la fuerza laboral en relación con la población total de 1955 a 1973), un cambio de excedente a escasez de la mano de obra (lo que ayudó a reducir la brecha en los salarios entre empresas grandes y pequeñas), ${ }^{15}$ más trabajadores en el sector manufacturero a expensas del sector agrícola, altos niveles de ahorro personal y de inversión, ${ }^{16}$ y sobre todo, la mayor escolaridad de la fuerza laboral.

Durante este primer periodo de la posguerra, el modelo familiar en Japón expresó los primeros rasgos de la clase media. Esposos asalariados con esposas dedicadas de tiempo completo a un hogar que todavía mos-

${ }^{13} Y$ Ytaka Kosai, “The postwar Japanese economy, 1945-1973”, en John W. Hall, Marius B. Jansen, Madoka Kanai y Denis Twitchett (coords.), The Cambridge History of Japan, 1988, vol. 6, Cambridge, Cambridge University Press, p. 495.

${ }^{14}$ Kelly, "At the limits", op. cit., p. 238.

${ }^{15}$ Kosai, op. cit., p. 510.

${ }^{16}$ Ibid., p. 508. 
traba tres generaciones bajo el mismo techo con la autoridad indiscutible del padre. En esta familia en proceso de cambio, las madres dedicadas al hogar comenzaron, poco a poco, con el paso de los años, a ser incorporadas a la fuerza laboral en fábricas y las jóvenes empezaron a dejar de prepararse para ser esposas y madres y a prepararse para la universidad. ${ }^{17}$ Una manifestación de la ampliación de la clase media fue la posibilidad de adquirir artículos electrodomésticos y, para la década de 1960, automóviles, todo gracias a mejores ingresos.

\section{Distribución de los ingresos}

La clave de la consolidación de una clase media en Japón parece haber sido una distribución del ingreso lo más homogénea posible. De acuerdo con Kosai, en los primeros años de posguerra aumentó considerablemente el ingreso de ciertos empresarios individuales (principalmente agricultores y comerciantes), al tiempo que disminuyó el porcentaje del ingreso nacional destinado a ingresos por salarios, ingresos por propiedad e ingresos corporativos. Esta situación cambió paulatinamente desde mediados de la década de 1950, y ya para la década siguiente, cuando se consolidó el periodo de crecimiento económico acelerado, aumentó el ingreso nacional proveniente de ingresos por salarios e ingresos corporativos al tiempo que disminuían los ingresos de los empresarios individuales. Este proceso redujo la brecha entre los salarios de los empleados y de los empresarios individuales y las empresas familiares. En esas dos décadas, sobre todo en la segunda, se perciben claramente tipos de empleo relacionados con la clase media, en particular de mandos medios en las empresas o profesiones derivadas de la educación universitaria, además de que aumentaron los trabajadores de "cuello blanco" en las principales urbes. ${ }^{18}$

${ }^{17}$ Suzanne Hall Vogel, "Japanese society under stress: Diagnosis and prescription”, Asian Survey, 52/4 (2012), p. 688.

${ }^{18}$ Kelly, "At the limits", op. cit., p. 236. 
Como vimos, luego de la guerra se produjeron cambios importantes en la estructura socioeconómica del país, impulsados originalmente por las autoridades de ocupación. La reforma agraria, la disolución de los zaibatsus y la reforma laboral ayudaron a homologar los ingresos en Japón. Durante los primeros años de la ocupación, el gobierno japonés encaró serios problemas económicos: se estima que la inflación de esos primeros años y el empobrecimiento de la población, junto con nuevos impuestos sobre la propiedad, llevaron a lo que Kosai señala como "igualdad de pobreza". ${ }^{19}$ Con la recuperación económica de mediados de la década de 1950, continuó el proceso de homologación en la sociedad, pero para entonces con mejores condiciones. En la década siguiente volvió a manifestarse esa tendencia por la reducción de los diferenciales salariales como resultado de que había más empleos.

\section{Sentimiento de pertenencia a la clase media}

Según estudios realizados por la Oficina del Gabinete japonés, la conciencia de pertenecer a la clase media pasó del $70 \%$ de la población en 1958 a casi el $90 \%$ a mediados de la década de $1960 .{ }^{20}$ Este gran segmento de la población se caracterizó por sus nuevos patrones de consumo de bienes de uso duradero (por esto son considerados también como una clase de consumo en masa), más afines al sistema universitario como mejor forma de favorecer la movilidad social y más propensos a ahorrar para asegurar el futuro de la familia. Este alto porcentaje de la población se sentía identificado con alguno de los tres estratos de clase media, principalmente clase media baja y clase media, aunque algunos de clase media alta, mientras que alrededor del 10\% entre finales de la década de 1950 y comienzos de la siguiente se identificaba con la clase baja. ${ }^{21}$ Por último, alrededor del $1 \%$ de la población se percibía como de la clase alta.

${ }^{19}$ Kosai, op. cit., p. 512.

${ }^{20}$ Chiavacci, op cit., p. 11; Kelly, "At the limits", op. cit., p. 235; Sawako Shirahase, "Japan as a stratified society: With a focus on class identification", Social Science Japan Journal, 13/1 (2010), p. 32.

${ }^{21}$ Chiavacci, op. cit., p. 11. 
Este proceso de consolidación de una conciencia de clase media, lento pero sostenido, pasó por una etapa preliminar durante la segunda mitad de la década de 1950. Chiavacci ${ }^{22}$ señala que en esa década, a pesar de la desconcentración de ingresos y los signos del surgimiento de una sociedad de consumo en masa, los conflictos sociales entre las poderosas uniones sindicales y la resistencia popular contra la renovación del Tratado de Cooperación y Seguridad entre Japón y Estados Unidos de 1960 mostraron que la sociedad de clase media todavía distaba mucho de ser una realidad.

Si se considera que en la década de 1960 se definió el crecimiento económico como el objetivo primordial del país (se propuso como objetivo duplicar el PIB), se detectan tres cambios importantes que promovieron la consolidación de esta identidad de la mayoría de la población con una clase media. En primer lugar, la reconstitución del grupo de poder conservador (el Partido Liberal Demócrata, PLD, en el poder desde mediados de la década anterior) con su defensa del llamado "crecimiento compartido". En segundo lugar, la creación del modelo japonés de empleo, concebido para armonizar y relajar las relaciones laborales que habían sido particularmente tensas desde las grandes huelgas de 1960. Este modelo consistió en ofrecer garantías de empleo vitalicio en la compañía o el sector público, establecer una estricta jerarquía de salarios y promociones, y estimular la cooperación entre el sector patronal y el laboral. En tercer lugar, la generalización del consumo en masa y un ascenso de la movilidad social como resultado del alto crecimiento y por medio de la educación universitaria. ${ }^{23}$ En esta consolidación de la conciencia de pertenecer a una clase media, es sabido que los medios masivos de comunicación tuvieron un papel importante.

En la década de 1970, se reafirmó esta conciencia, ${ }^{24}$ y parte del éxito radicó en la confianza en la educación superior como canalizador de la movilidad social. Así, Japón aparecía como un país donde el éxito

${ }^{22}$ Loc. cit.

${ }^{23}$ Ibid., p. 12.

${ }^{24}$ A pesar de que ya en 1971 Vogel había identificado importantes dinámicas y cambios en esta clase media en términos de movilidad y aspiraciones personales de los asalariados. Vogel, op. cit., p. 273. 
educativo del individuo era condición para ascender en la sociedad. El gobierno reforzó esta imagen de sociedad igualitaria y justa, "para complementar el discurso de la particularidad cultural de Japón”. ${ }^{25}$ La imagen del asalariado (sarariman) se convirtió en modélica: un empleado de una gran compañía o de la burocracia gubernamental que pasó por la escuela media básica en academias de regularización (juku) y se preparó en escuelas de educación media superior para aprobar los exámenes de admisión y entrar a una universidad, a cuyo término le espera un empleo vitalicio con el que pueda llevar una existencia desahogada. Mientras tanto, la mujer seguiría el modelo de las "madres educadoras" (kyoiku mama) para transmitir a sus hijos este mismo ideal de vida. La aplicación y aceptación de este paradigma pudo hacerse gracias a que aumentó el número de empleos de oficina.

Esta idea de Japón como una sociedad de credenciales, donde para lograr el éxito personal y social era necesario graduarse de alguna universidad de prestigio, se mantuvo hasta el fin de la burbuja económica a inicios de la década de 1990. Para finales de la era Showa (1926-1989), el país se había consolidado como una meritocracia medida en términos de los logros y el prestigio educativo. ${ }^{26} \mathrm{Al}$ mismo tiempo, hasta esa década de 1990 la tradicional vida rural japonesa dio paso entre la creciente clase media a una existencia urbana en apartamentos, lo cual tuvo enormes implicaciones para las dinámicas de parentesco, incluyendo la atomización de la familia. Entre 1970 y 2005, los hogares multigeneracionales disminuyeron de $16.1 \%$ a $6.9 \%$, los hogares nucleares se mantuvieron entre el $56 \%$ y el $61 \%$, y aumentaron de $20.3 \%$ a $29.5 \%$ los hogares compuestos por una sola persona. ${ }^{27}$

\section{Cambios desde la década de 1990}

Sugimoto afirma que el sentimiento de gran parte de la sociedad japonesa de ser parte de una clase media podría ser una manifestación de pertenencia social que esconde, en contraste, un sentimiento intenso

${ }^{25}$ Ibid., p. 13.

${ }^{26}$ Kelly, "At the limits", op. cit., p. 240.

${ }^{27}$ Vogel, op. cit., p. 288. 
de desigualdad socioeconómica, un sentimiento no expresado por acatar ciertos principios formalmente establecidos. ${ }^{28}$

En esta década inició un proceso reversible en el que Japón comenzó a identificarse como una sociedad dividida (kakusa shakai) según un nuevo modelo de desigualdad social, o como Kelly señala, por la aparición de "nuevos ricos" y "nuevos pobres". ${ }^{29}$ Investigadores como Ishida Hiroshi y Kanomata Nobuo señalan que desde mediados de la década de 1980 las posibilidades de ascenso laboral han disminuido y la desigualdad en los ingresos se ha ampliado. ${ }^{30}$ Esto mismo pareció ocurrir en otras naciones de la OCDE, que en esos años mostraron tendencias similares de desigualdad salarial creciente. En el caso de Japón, donde desde mediados de la década de 1970 se ensanchó la brecha de los ingresos, ${ }^{31}$ diversos factores han puesto de relieve este cambio, como el envejecimiento de la población, el alto número de jóvenes que viven solos, el bajo salario ofrecido a los recién graduados, etc. Otros factores que influyeron en este cambio paradigmático fueron la liquidación de la burbuja económica a inicios de la década de 1990, cambios en el sistema internacional, la propia muerte del emperador Showa Hirohito en 1989 y las crisis políticas por un cambio de poder en 1994 que terminó brevemente con un régimen largo e ininterrumpido del hegemónico Partido Liberal Demócrata. A esto hay que sumar la fase inicial de estancamiento económico y deflación de la economía (al término de la burbuja económica) que coincidió con la recuperación económica de Estados Unidos. Ante estas duras condiciones económicas, la percepción de la recuperación y la competitividad internacional japonesa habría quedado condicionada al abandono de un modelo igualitario y a favor de salarios según los méritos.

En relación con la cuestión de los ingresos familiares, se ha visto una reducción del promedio de los ingresos por familia. En esa década

${ }^{28}$ Sugimoto, op. cit., pp. 26, 33.

${ }^{29}$ Kelly, "At the limits", op . cit., p. 233. Sugimoto considera, sin embargo, que la aceptación de este cambio en Japón desde esa década, y no antes, ha sido objeto de un animado debate. Yoshio Sugimoto, "Class and work in cultural capitalism: Japanese trends", The Asia-Pacific Journal, 8/40 (2010), p. 2.

${ }^{30}$ Chiavacci, op. cit., p. 18.

${ }^{31}$ Kelly, "At the limits", op. cit., p. 233. 
de 1990, los jóvenes recién graduados comenzaron a tener problemas para encontrar un puesto de trabajo, y ahora muchos empleos son de medio tiempo o por contrato, sin beneficios y pensiones. El empleo vitalicio (shushin koyou) y la estricta jerarquía indispensable para determinar ascensos tienden a perderse. Si bien Sato explica que el porcentaje de trabajadores regulares ha ido al alza desde $1985,{ }^{32}$ Vogel señala que la proporción de trabajadores no regulares aumentó de ser el $20.2 \%$ del total de la fuerza laboral en 1990 a ser el 33.7\% en el año $2010 .{ }^{33}$ Este cambio drástico en el mercado laboral tiene su correlato en el índice de desempleo, que se ha elevado en la última década. En 2004, la tasa de desempleo entre los adolescentes era de más del $30 \%$ para los que no terminaron la educación media superior y del 15\% para los graduados. ${ }^{34}$ En 2011, según cifras del Ministerio del Trabajo japonés, solo el 68.8\% de los egresados de una universidad y la mitad de quienes acabaron estudios técnicos superiores encontraron trabajo. ${ }^{35}$ Terminar la educación media superior o la universidad ya no garantiza un empleo; si se consigue, puede no ser de tiempo completo y es muy difícil que sea de por vida. Lo anterior ha provocado un "desempleo voluntario" entre los universitarios recién graduados: están en busca de la oportunidad adecuada para obtener un trabajo propicio para su futuro en las grandes empresas o el gobierno, y desechan las ofertas laborales en compañías medianas o pequeñas.

Desde comienzos del siglo XXI, parecería que el tradicional modelo de clase media de la posguerra fue remplazado por el de una sociedad desigual. En el análisis sociológico priva ahora un discurso que integra exámenes y descripciones de los trabajadores pobres, independientes (furitia), ninis japoneses y elementos antisociales, al tiempo que se vuelven más marcadas las diferencias entre la población de las urbes

${ }^{32}$ Yoshimichi Sato, "New middle class at risk in Japan?", ponencia presentada en la 106 Reunión Anual de la Asociación Estadounidense de Sociología, Las Vegas, 20-23 de agosto de 2011, p. 4.

${ }^{33}$ Vogel, op. cit., p. 688.

${ }^{34}$ Rebick, op. cit., 187.

${ }^{35}$ Yoko Wakatsuki, "Hiring of Japanese grads drops to a record low", CNN, 18 de enero de 2011, en <http://edition.cnn.com/2011/WORLD/asiapcf/01/18/japan.graduate.jobs/>, consultado el 15 de febrero de 2018. 
y del campo. David Slater señala que en el sector laboral, esta "nueva clase trabajadora" se caracteriza por estar integrada por trabajadores ocupados en el sector de servicios, con poca estabilidad y pocos beneficios, un tipo de trabajo característico del nivel más bajo del mercado laboral, al igual que en muchas economías postindustriales. ${ }^{36}$ En el extremo, y particularmente visible ahora en grandes urbes como Tokio, está el fenómeno de personas sin hogar que, por diversos motivos como deudas, desempleo o incluso deshonor familiar, vagan por parques y calles. Este fenómeno contrasta con una nueva clase de ricos que presumen niveles de vida representados en los medios masivos de comunicación y que no ocultan su riqueza al pasearse y gastar en lujosas tiendas de los distritos comerciales de Ginza y Omotesando de la capital.

En el plano político, las reformas económicas del primer ministro Junichiro Koizumi (2001-2006) y sus predecesores parecen haber tenido un alto costo, pues profundizaron la desigualdad social y ensancharon la brecha de los ingresos. En particular, la reubicación de muchas empresas en el extranjero y el aumento del impuesto al consumo han afectado a la población en general, pero particularmente al sector laboral. Asimismo, como resultado de la mayor liquidez — parte de las tres flechas de los Abenomics-, se han incrementado las ganancias bursátiles del minúsculo grupo de ricos del país. De hecho, la desigualdad social parece haber sido uno de los principales temas que propiciaron desde 2007 la resistencia de la sociedad japonesa al primer gabinete de Shinzo Abe.

Uno de los principales cambios de las familias es que los hijos ya no tienen garantizado un puesto vitalicio en una empresa luego de graduarse de una buena universidad. Los jóvenes recién graduados se conforman con empleos que no corresponden al prestigio que antaño implicaba graduarse de una universidad de prestigio. Esta "conmoción laboral" (jobbu shocck) ha dañado la confianza de estas generaciones en su futuro. Kelly cita al sociólogo Hishida Hiroshi, quien entiende que en los últimos treinta años, obtener una educación elitista en Japón ha dejado de tener un efecto de movilidad social significativo. ${ }^{37}$ Se atri-

${ }^{36}$ Slater, op. cit., p. 4.

${ }^{37}$ Kelly, "At the limits", op. cit., p. 249. 
buye la poca movilidad social a cambios estructurales (entre ellos, un nuevo equilibrio entre la demanda de empleo en las empresas y el nivel educativo universitario masificado) que se manifestaron al final del milenio. ${ }^{38} \mathrm{Al}$ mismo tiempo, los jóvenes abandonan el hogar de los padres y los ancianos ya no viven con sus hijos adultos. ${ }^{39}$

En relación con las principales consecuencias de esta nueva realidad, hay que señalar que el deterioro en los empleos parece obligar cada vez más al padre y a la madre a trabajar, retarda la decisión de tener hijos $\mathrm{y}$, entre la población soltera, lleva a considerar el matrimonio como secundario. Para reforzar el índice de natalidad y la permanencia de las madres trabajadoras en sus puestos de trabajo, el gobierno ha tomado medidas radicales, como, por ejemplo, la decisión de promulgar en 1985 la Ley de Igualdad de Oportunidades Laborales, los Planes Ángeles, promulgados en 1995-97 y 2004 para aumentar el número de guarderías, así como la Ley de Seguro del Empleo de 1994-95, para garantizar 10 meses posparto de licencia pagada por maternidad..$^{40}$

Mientras tanto, la inseguridad de mantener un empleo permanente debido al cambio de las políticas de contratación ha llevado a que las mujeres, cada vez más presentes en la plantilla laboral del país, ocupen más empleos temporales. Este dato se considera un factor importante para los cambios graduales en el índice de Gini y en la tendencia a la desigualdad: en la sociedad japonesa, el salario de las mujeres es en general menor al de los hombres para funciones similares, y es bien sabido que reciben menos beneficios. ${ }^{41}$

Por otro lado, el ingreso de más mujeres al mercado laboral ha producido cambios en las preferencias de contraer matrimonio y tener hijos. Kelly señala que los varones japoneses enfrentan desde la década de 1980 una verdadera crisis de matrimonio, mientras que en las mujeres se han colapsado las tasas de fertilidad. Estos dos factores han sido decisivos para la baja de la población. ${ }^{42}$ Son cada vez más numerosas las mujeres

\footnotetext{
${ }^{38}$ Rebick, op. cit., 184.

${ }^{39}$ Vogel, op. cit., p. 694; Kelly, “At the limits”, op. cit., p. 243.

${ }^{40}$ Vogel, op. cit., p. 700.

${ }^{41}$ Rebick, op. cit., 183.

${ }^{42}$ Kelly, "At the limits", op. cit., p. 245.
} 
que cruzan solteras el límite seguro de reproducción (aproximadamente a los 35 años) y que no tienen mucho interés en formar una familia. Más aún, si una trabajadora decide vivir sola, es más probable que además reciba un ingreso bajo. ${ }^{43}$ Las mujeres comparten este destino con los hombres menos instruidos: ambos grupos ganan menos que el grupo de hombres con educación universitaria. ${ }^{44}$

\section{Reflexiones sobre las causas actuales de la desigualdad en Japón}

Una de las manifestaciones más visibles de la desigualdad social en Japón, vinculada con la desigualdad de ingresos, es la brecha entre los jóvenes y su siguiente generación. Sawako Shirahase, ${ }^{45}$ utilizando encuestas de opinión pública sobre estilo de vida de la Oficina del Gabinete, realizó un importante estudio sobre identidad de clase y desigualdad, y concluyó que desde finales de la era Heisei (1989) hasta alrededor de 2005, el estancamiento económico ha afectado marcadamente a jóvenes de entre 20 y 34 años y los ha dejado en una posición vulnerable en el mercado laboral.

Esta vulnerabilidad no tiene que ver únicamente con el desempleo, sino también con la precarización del trabajo. Estimaciones del Ministerio del Trabajo de finales de 2015 ubican el salario promedio mensual de un trabajador de medio tiempo en 96638 yenes (unos 800 dólares), mientras que el salario promedio mensual de un trabajador de tiempo completo era de 352094 yenes (equivalentes a 2900 dólares) ${ }^{46}$ A partir de la primera mitad de la década de 1990, ha disminuido el promedio de los salarios de tiempo completo, como se muestra en la siguiente gráfica:

${ }^{43}$ Rebick, op. cit., 183.

${ }^{44}$ Sato, op. cit., p. 3.

${ }^{45}$ Shirahase, loc. cit.

${ }^{46}$ Reiji Yoshida, "Critics assail Abe after middle-class income gaffe highlights wealthy upbringing", The Japan Times, 9 de enero de 2016, en <http://www.japantimes.co.jp/ news/2016/01/09/national/politics-diplomacy/critics-assail-abe-middle-class-income-gaffehighlights-wealthy-upbringing/\#.WRxh8dylupo>, consultado el 15 de febrero de 2018. 


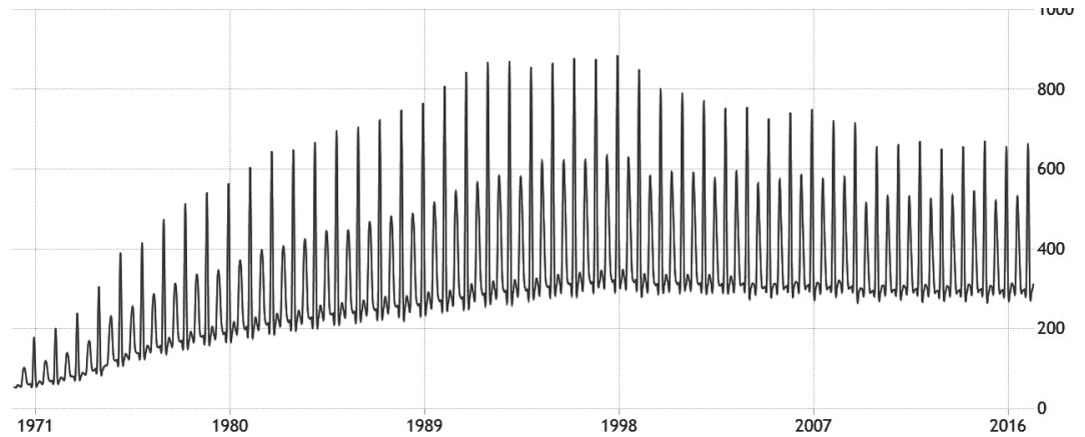

Fuente: Trading Economics (2017), <http://www.tradingeconomics.com/japan/wages >

Diversos factores hay moldeado la brecha actual de los ingresos, que es la causa principal de la percepción de desigualdad social y el sentimiento de precariedad entre la clase media japonesa. En primer lugar, hay factores empresariales, como la diversidad de las compañías, el número de empleados y el tipo de empleo ofrecido. La diferencia entre las grandes empresas y el creciente número de compañías pequeñas y medianas, así el tipo de contratos otorgados a los trabajadores, ha desembocado en las actuales prácticas laborales. Los beneficios otorgados a los oficinistas según los principios de edad y antigüedad, así como el empleo vitalicio, se ofrecen solo a los empleados regulares y principalmente de grandes empresas, por lo que "el tipo de empleo y el tamaño de la empresa ejercen una fuerte influencia en la estratificación social". ${ }^{4}$ Atsushi Sannabe ${ }^{48}$ calculó que, si en 1984 el porcentaje de varones trabajadores de medio tiempo era del $8 \%$ de la fuerza laboral total, este porcentaje subió al 19\% en 2010 .

Esta situación se ha agravado debido a la modificación de la pirámide demográfica en un país en el que hay cada vez más personas de la tercera edad y menos jóvenes. En las primeras décadas de la posguerra, un nutrido número de empleados jóvenes en las empresas consi-

${ }^{47}$ Shin Arita, "A comparative analysis of social stratification in Japan, Korea and Taiwan: Where is the locus of social inequality?", ISS Discussion Paper Series, (febrero, 2017), en $<\mathrm{http}: / /$ www.iss.u-tokyo.ac.jp/publishments/dpf/pdf/f-182.pdf>, consultado el 15 de febrero de 2018.

${ }^{48}$ Atsushi Sannabe, "Occupational inheritance: Impact on long-term worklessness and unemployment, human networks, and happiness", Japan Labor Review, 12/3 (2015), p. 54, en <http://www.jil.go.jp/english/JLR/documents/2015/JLR47_sannabe.pdf>, consultado el 15 de febrero de 2018. 
deraban que en el futuro tendrían una buena oportunidad de ascenso cuando alcanzaran la edad suficiente para subir a los mandos medios o altos. Ahora son cada vez más peleados estos puestos por la gran oferta de trabajadores de cierta edad. Rebick señala que se ha vuelto menos probable la promoción laboral y, con ello, la movilidad social y el aumento de salario. ${ }^{49}$ Sin embargo, en algunos estudios se indica, como señala Sato, que la práctica del trabajo vitalicio persiste en las grandes empresas, a diferencia de las pequeñas y medianas. ${ }^{50}$ En otras palabras, todavía se protege el empleo vitalicio de los trabajadores de tiempo completo, mientras que en los demás casos, el puesto es irregular o de medio tiempo.

Otros factores están relacionados con el sistema educativo. Es conocida la gran dificultad de aprobar el examen para ingresar a las universidades más prestigiadas del país, pero ahora incluso un título de estas instituciones no garantiza un buen empleo a largo plazo. Más aún, incluso un trabajador educado en una universidad promedio, corre el riesgo de cambiar su estatus laboral de regular a irregular. Es difícil el ascenso intrageneracional hacia un estatus mejor, además de que se corre el riesgo de ver cambiar un empleo vitalicio por uno irregular $\mathrm{y}$, con ello, ver cernirse la sombra de la pobreza.

En un Japón cada vez más globalizado, el factor educativo incide en la competitividad laboral internacional y se vincula con factores institucionales que tienen implicaciones en los trabajadores y en sus familias. Como debido a las reformas estructurales es cada vez más difícil la movilidad social para un trabajador, muchas personas buscan soluciones por cuenta propia con implicaciones a largo plazo. Rebick explica que el nivel de autoempleo de los trabajadores retirados era alto en la década de 1980, pero que actualmente es muy difícil de prosperar: por un lado, las reformas han retirado beneficios a los pequeños emprendedores (que montaban su propia empresa y aprovisionaban a su antiguo patrón), y por el otro, muchas actividades empresariales se subcontratan en otros países, principalmente el sudeste de Asia, que tienen costos más bajos.

${ }^{49}$ Rebick, op. cit., 186.

${ }^{50}$ Sato, op. cit., p. 3. 
Así, el aspirante a tener un empleo propio enfrenta precariedad en dos frentes. ${ }^{51}$

Durante los últimos años, en particular como resultado de la lenta recuperación económica de la crisis financiera de 2008, los trabajadores han encontrado diversas formas de hacer frente a su pauperización y a las reformas que han dado al sector empresarial más flexibilidad en las relaciones contractuales. Ahora prolifera el empleado independiente, apoyado en el avance de las tecnologías de la información, que es de hecho una fuerza laboral paralela al empleado regular de una empresa. En la actualidad, en Japón hay poco más de 11 millones de independientes, lo que representa el 17\% de la población económicamente activa del país. ${ }^{52}$

En dirección contraria a la cultura tradicional de empleo, la antítesis del sistema de inicios de la posguerra, una comisión de reforma laboral del gobierno japonés presentó a principios de 2017 un informe en el que recomendaba mantener "estilos de trabajo flexibles" que promuevan el trabajo independiente subrogado, incluyendo las horas extras fuera del horario laboral regular. Si bien el trabajador puede ganar buenos ingresos en la medida en que contrate trabajos, en realidad solo agravará su situación laboral para el futuro: al no ser miembro de una empresa, deja de ser sujeto de crédito en instituciones financieras, es discriminado por los empleados regulares, cae presa de intermediarios y no percibe diversos beneficios y seguridad social, entre otras desventajas. ${ }^{53}$

\section{Conclusiones}

Probablemente es un error preguntar si Japón ha dejado de ser la sociedad de clase media que fue concebida a inicios de la posguerra. Si bien no hay datos para calcular hoy el porcentaje total de personas que se

${ }^{51}$ Rebick, op. cit., 185.

${ }^{52}$ Shusuke Murai, "Freelancing shines for flexibility but dark side awaits", The Japan Times, 14 de mayo de 2017, en $<$ http://www.japantimes.co.jp/news/2017/05/14/national/can-japan-landof-lifetime-employment-handle-the-rise-of-freelancers/>, consultado el 15 de febrero de 2018.

${ }^{53}$ Ibid. 
perciben como miembros de la clase media (la encuesta SSM dejó de compilarse en 2005), y aunque puede asumirse que sigue siendo mayoría, la naturaleza de esta clase media es radicalmente distinta de la que hubo entre las décadas de 1960 y 1990. En Japón, la población considerada de clase media tiene cada vez más problemas para mantener la confianza en el futuro, además de que también parece haber una crisis de identidad, de pertenecer a esa clase media: Akio Doteuchi, del Instituto de Investigaciones DLI, señala que la amenaza principal proviene del riesgo de caer en la pobreza si ocurre algo inesperado en la vida del trabajador o de su familia, como una enfermedad costosa, la incapacidad de obtener un buen empleo al salir de la universidad o tener que ocuparse de un familiar de la tercera edad. ${ }^{54}$ El peligro es bastante real, según las estadísticas: a inicios de 2015, el total de hogares en pobreza que dependían del sistema nacional de bienestar era de 1618 817, una cifra que vino aumentando en las últimas dos décadas. ${ }^{55}$

Al mismo tiempo, el avance en las reformas económicas, resumidas en los Abenomics del primer ministro Shinzo Abe, vaticina ajustes en las condiciones del mercado laboral y la continuación de la baja en los costos laborales. Lo más probable es que esas condiciones laborales sigan siendo precarias. Las grandes empresas todavía pueden garantizar empleo vitalicio a sus mandos medios y ejecutivos, para que gocen de los beneficios del tradicional sistema laboral de la posguerra, los trabajadores poco capacitados están — como señala Sato_ “a la deriva en la creciente inestabilidad del mercado laboral", ${ }^{56}$ caracterizada por la desregulación y la tendencia hacia el empleo temporal.

Entonces, la pregunta es cómo paliar la disparidad económica en la sociedad japonesa. Una de las posibles soluciones es que el gobierno intente acortar la brecha de los salarios entre los trabajadores regulares y los irregulares, aunque las más recientes políticas para combatir el

${ }^{54}$ Hiroko Nakata Hiroko, "Under 'Abenomics,' rich thrive but middle class on precipice", The Japan Times, 7 de abril de 2015, en <http://www.japantimes.co.jp/news/2015/04/07/business/economy-business/abenomics-rich-thrive-middle-class-precipice/\#.WRt4pdy1upo>, consultado el 15 de febrero de 2018.

${ }^{55}$ Loc. cit.

${ }^{56}$ Sato, op. cit., p. 10. 
desempleo se dirigen en dirección contraria, hacia la promoción de empleos temporales, de medio tiempo o en esquemas de ingresos extras. Por otro lado, y considerando que la brecha de salarios es eminentemente generacional, el gobierno debe ayudar a los jóvenes a encontrar un empleo estable. De que se encuentren las respuestas depende el futuro de una ahora precaria clase media amenazada por las fuerzas de la globalización, por el envejecimiento de su propia población y por las reformas estructurales diseñadas desde las élites del poder. 
Se prohíbe su reproducción total o parcial por cualquier medio, incluido electrónico, sin permiso previo y por escrito de los editores. 